\title{
EL TURISMO ES LA GUERRA POR OTROS MEDIOS. LA BIOPOLÍTICA Y GEOPOLÍTICA DEL DESTINO CANARIAS
}

\author{
Pablo Estévez Hernández* \\ Escuela Superior de Turismo Iriarte
}

\section{RESUMEN}

La historia de Canarias refleja un pasado donde la inclusión en el proyecto de la modernidad provocó una articulación espacial que recrea espacios en una tensión entre lo local y lo global, o, en otras palabras, entre las biopolíticas y geopolíticas que generan el espacio. Como validación de esa instancia en la modernidad, Canarias ha pasado por distintas etapas socioeconómicas, culminando con la industria del turismo, que concibe el espacio no de manera autónoma, sino siguiendo nuevas nomenclaturas que enganchan con un imaginario colonial y exótico, al tiempo que altamente regulado.

Palabras Clave: turismo, biopolítica, Islas Canarias, riesgo.

\author{
TOURISM IS WAR BY OTHER MEANS. \\ THE BIOPOLITICS AND GEOPOLITICS \\ OF THE CANARY ISLANDS DESTINATION
}

\section{Abstract}

The history of the Canary Islands reflects a past where the inclusión in the project of modernity caused a spatial articulation that recreates spaces in a tension between the local and the global, or, in other words, between the biopolitical and geopolitical tropes that generate that space. As a validation of that instance in modernity, the Canary Islands has gone through different socioeconomic stages, culminating with the tourism industry, which conceives space not autonomously, but following new nomenclatures that engage with a colonial and exotic imaginary, though highly regulated.

Keywords: tourism, biopolitics, Canary Islands, Risk. 
La isla más isla de todas las islas es la inaccesible, la isla a la que nunca se puede llegar. Isla es parador y tregua en la inmensidad de las aguas pavorosas; es jalón y remedo de tierra firme. Tierra firme ha sido siempre tierra en serio, continente y no esa angustia de trozos, fragmentos de verdad, que son las islas.

María Rosa Alonso Rodríguez. San Borondón, signo de Tenerife.

Me muero de ganas de volver a mi hogar, y desde luego ya no está aquí. Nah, para mí son las Canarias: sol todo el año y chochitos que vienen de vacaciones y se les cae la baba de ganas. Por mi Inglaterra se la pueden meter en el puto culo.

Irvine Welsh. Si te gustó la escuela, te encantará el trabajo.

\section{CANARIAS: MODERNIDAD Y TURISMO}

Este ensayo investiga las relaciones espaciales que se dan en las Islas Canarias bajo distintos paradigmas sobre ordenación y fórmulas para presentar el territorio (en la biopolítica y la geopolítica) con atención específica a un proceso de modernización (el turismo) ${ }^{1}$. Estos representan distintas maneras de contener el espacio en nomenclaturas que obedecen a escalas o lógicas de la economía global, imbricadas en historias que se mueven por distintos delineamientos modernos. Por querer resaltar estos dos aspectos, que pueden obedecer a distintas figuras de imperio global, de soberanías aplicadas como fórmulas de gobierno y control a distancia, se debe recaer también en las resistencias que han provocado que ningún discurso acerca de la realidad territorial y geopolítica de Canarias quede enclaustrado en una taxonomía definitiva o en un orden al lado de la balanza mundial que queda resquebrajada por la diferencia colonial (véanse distintas intervenciones sobre este concepto en Castro-Gómez y Grosfoguel, 2007). El resultado de todo esto es que el único dominio claro sea el de la ambivalencia.

Insertada en la modernidad desde la Conquista (un conjunto de proyectos privados y estatales que duró desde 1402 a 1499) ${ }^{2}$, Canarias se fue gestando como un punto de paso para distintos agentes operativos en proyectos imperiales y comerciaEspaña).

* E-mail: pabloestevez4@gmail.com. Escuela Superior de Turismo Iriarte (Islas Canarias,

${ }^{1}$ Este ensayo se redactó antes de la crisis sanitaria provocada por la covid-19, por lo tanto, las observaciones de la configuración espacial del destino pueden verse alteradas con las nuevas disposiciones políticas y sanitarias en un destino turístico que pretende seguir los patrones de consumo de ocio en un mundo postpandémico. En cualquier caso, las disquisiciones teóricas sobre la biopolítica y la geopolítica como la aquí propuesta serán de primer orden para el estudio de la gestión pública y privada del turismo en un lugar como las Islas Canarias.

${ }^{2}$ La teoría decolonial traza una genealogía de la modernidad solo marcado por la Ilustración como movimiento cultural y a la revolución industrial como motor económico, sino que la piensa con su anverso: la colonialidad. En este sentido, el colonialismo moderno da lugar a la imagen moderna en el centro europeo. En consecuencia, la modernidad nace con el establecimiento del sistema-mundo moderno colonial, con el hito de Colón y el nacimiento del entramado atlántico figurando como precursores en la genealogía de esta modernidad/colonialidad (véase, entre otros, Castro-Gómez y 


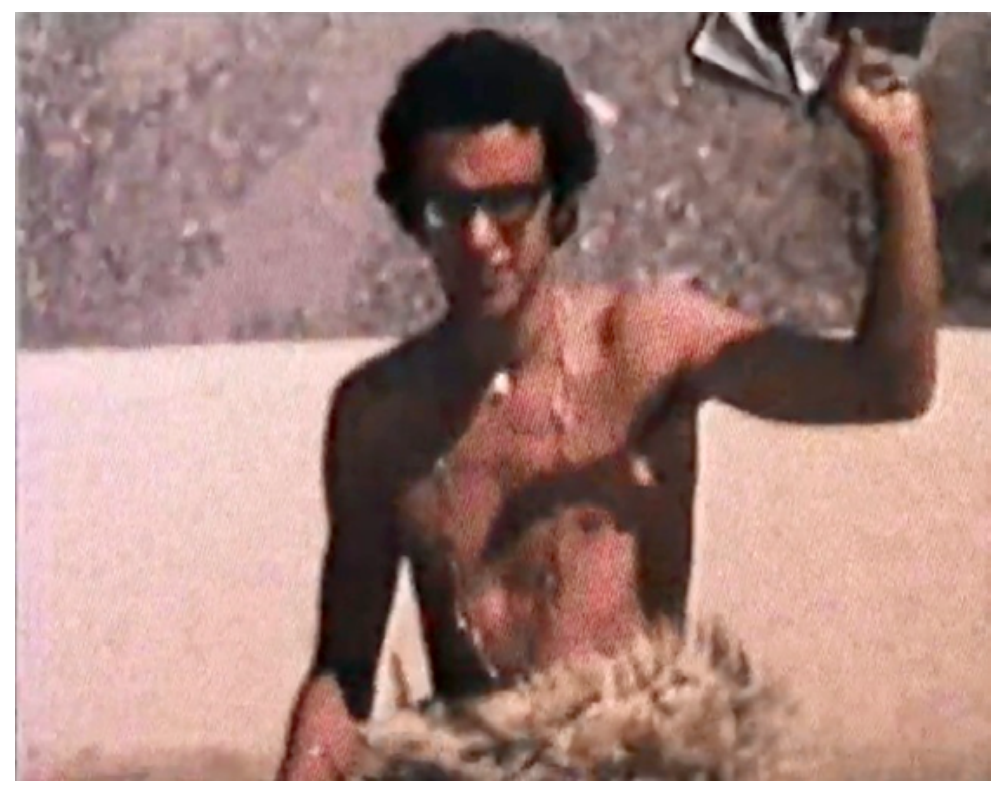

Fotograma del film Crónica histérica. La conquista de Tenerife. Equipo Neura (1974).

les, que incluyeron el tráfico de esclavos y el transporte de mercancías. Es por tanto que las Islas se convirtieron en un territorio atravesado por la violencia en la consolidación de una sociedad criolla, que empezaba a aunar sus componentes desde la culminación del proceso de Conquista, en el momento mismo del bautismo de los últimos guanches (indígenas canarios) capturados en Tenerife.

Esta nueva sociedad podría considerarse una "zona de contacto», en la terminología de Mary Louise Pratt (1996) ${ }^{3}$, donde se encuentran físicamente cuerpos diversos que realizan viajes y que traen consigo toda una serie de materiales e ideas que los acompañan, viajando a su vez en "ciudades flotantes», tal como denominaba el escritor Alonso Quesada a los barcos. Al primer encuentro colonial entre europeos e indígenas le siguieron una serie de encuentros que se tornarían como una especie de eterno retorno de aquel original. Ilustrando irónicamente el primer encuentro, un equipo de cineastas canarios en los ańos setenta, usando cámaras super- 8 para

Grosfoguel, 2007). Para algunas aproximaciones de esta teoría en Canarias véanse los trabajos de Larisa Pérez Flores (2017) y Roberto Gil Hernández (2015).

3 «Las zonas de contacto tienen con frecuencia su origen en la invasión y la violencia y se traducen en formaciones sociales que se basan en drásticas desigualdades. A menudo también entrañan lo que se ha llamado 'heterogeneidad radical', es decir, estructuras sociales en las que, en un mismo espacio, coexisten sistemas culturales muy diferenciados que interactúan entre sí» (Pratt, 1996, p. 7). 
grabar su propio cine independiente, rodaron el encuentro de manera alcoránica en una neoplaya canaria, tratando a los invasores castellanos como turistas a los que hay que venderles souvenirs nada más darles la bienvenida (Crónica histérica, Equipo Neura, 1974).

El turismo es el último gran proyecto que se ha insertado en las Islas ${ }^{4}$. Es, por tanto, el programa con el cual actualizamos la modernidad. El turismo suele ser visto como un modelo o un sector, pero en un espacio como son estas islas, el turismo es «algo más». Las relaciones espaciales y las nomenclaturas, que se tratarán como cronotopos en este texto, estarán contenidas en esta actualización. Por lo tanto, es el turismo como proyecto moderno en Canarias lo que se va a estudiar, no solo como un prisma para ver esos contenidos y estudiarlos, sino como un proceso que recrea el espacio mismo. El turismo tiene una incidencia enorme sobre cómo pensamos el espacio en los llamados destinos (una nomenclatura común).

Hay que señalar que en Canarias se ha elaborado una profusa literatura desde las ciencias sociales para el tratamiento del turismo. No es de extrańar, puesto que la misma percepción del territorio como destino ha provocado que la atención académica gire en torno a diversos aspectos del turismo (cuestiones importantes como su impacto, la estructura de género, las relaciones económicas, etc.). No obstante, siempre ha existido una metodología concreta que asienta al turismo como un objeto delimitable, incluso en un ámbito como Canarias, normalmente identificado como un sector, que puede aproximarse con la cuantificación de ciertos aspectos, pero que se retracta del estudio de sus ensambles.

El turismo aparece como un sistema coherente que informa las acciones que se dan en un espacio determinado (normalmente entre elementos estáticos y dinámicos que dan lugar al impacto). En este sentido, el espacio, o las relaciones espaciales, aparecen como nociones laxas o como meros contenedores de las acciones o las relaciones interpersonales y el intercambio económico que se da con el fenómeno5. En términos sociológicos se habla de estas mismas relaciones, pero incorporando

${ }^{4}$ También se ha hablado de la posibilidad de tipificar el turismo como una forma indistinguible de los procesos de extracción colonial/capitalista, como turismo poscolonial propiamente dicho:

... tourism is barely distinguishable from other forms of colonial economies. Typically founded by resident proto-entrepreneurs, the industry expands beyond institutional control, becomes institutionalized by large-scale forces of capital, and then grows to mirror not the values of the place but those of the travelling public (Rothman, 1998, p. 16).

5 Desde el ámbito de la llamada geografía postestructural o de la teoría de la no representación (Thrift, 2008) se ha considerado el estudio del espacio no como un mero contenedor de las interacciones humanas, sino que se ha centrado en la producción de espacios como un proceso relacional, donde los humanos implicados en las relaciones y el espacio se constituyen mutuamente. Esto acarrea una noción de un sujeto corporizado, y no una noción estándar de actante humano desligado de su entorno (para un análisis exhaustivo de esta perspectiva y sus principales aportaciones véase Murdoch, 2006). Desde el ámbito del marxismo, hace tiempo que el geógrafo David Harvey concibió las relaciones espaciales como una de las principales soluciones que el capitalismo tiene para operar su lógica acumuladora (Harvey, 2000, p. 23), deslocalizando siempre sus formas de producción, pero fomentando también espacios de esperanza (Harvey, 2000). 
matices sobre la clasificación de los turistas y los componentes sociales que puedan producirse en ese espacio. Cuando no, el concepto de destino parece tener problemas para ajustarse a lo entendido geográficamente como un territorio (Barrado Timón, $2004)^{6}$. Este sistema coherente parece darse en espacios carentes de sentido, vacuos y sin historia. Mucho menos suele aparecer una teoría del poder asociada al destino (Cheong y Miller, 2000).

Este ensayo examina las relaciones espaciales, pero tomando estas como dinámicas; procesos que involucran redes de actantes y una genealogía que precisa de los anversos de la modernidad y de la procreación de estas islas como satélites de Europa, con su espacio de ambivalencia marcando todas las dimensiones. Por último, intentará no limitar la localización de Canarias, sino de ver la interacción global que tiene este espacio en un mundo dominado por los riesgos globales modernos, mediatizados por expertos a escala planetaria (Beck, 2006). Sin duda, un paradigma del orden social que articula las sociedades y que se acerca a la planificación turística como una forma de gestión pospolítica. Comenzaré por ver la genealogía de estos procesos y los cambios de paradigma que se han dado en la constitución de un turismo que ha dominado la escena en los últimos años: el turismo de masas.

\section{DEL TURISMO COLONIAL AL POSCOLONIAL}

Podríamos definir el momento del turismo actual en Canarias como un turismo poscolonial. Sin duda, esta fórmula permitiría no solo comprender el turismo de masas, sino las peculiaridades del posturismo y las otras tantas modalidades practicadas en las Islas. Al mismo tiempo esto nos permitiría vincular algunas prácticas con relaciones turísticas en el pasado. Si existe un turismo poscolonial es, precisamente, porque hubo un turismo colonial. Aquí es donde se sigue recreando la representación del encuentro colonial (del «mítico primer encuentro»; Minca, 2009, p. 92) 7 , que ha pasado a cambiar el origen social y cultural de los que escenifican este

${ }^{6}$ No existe una definición estable de destino. Los marcos usados tradicionalmente desde la geografía no reconocían los espacios turísticos, categorizándolos dentro de otra nomenclatura más «tradicional»: suelo urbano/rural. Por lo tanto, las zonas turísticas respondían a la categoría de urbano, con algunas matizaciones.

Con estos presupuestos, el principal problema generado por la exclusiva atención al suelo urbano-turístico es que se desligó interpretativamente el espacio urbanizado del resto de las formas espaciales que el turismo necesita y genera, y que conforman un sistema inseparable con el espacio ocupado por los alojamientos y las infraestructuras (Barrado Timón, 2004, p. 47).

7 "Islands and their beaches are also, in a postcolonial perspective, mythical spaces of 'the first encounter'. 'The Island' is a geographical trope where the (physical) detachment from the mainland apparently allows for an entirely different set of perspectives, possibilities, identities, temporalities and spatialities. The Island embodies metaphorical and material separation from home: it appears as a clearly bounded geographical feature, delimited by the 'natural frontiers' of the water that surrounds it. Tourist islands, especially small tourist islands, are thus very often presented as microcosms, self-enclosed universes characterized by a highly simplified geography» (Minca, 2009, p. 92). 
encuentro. El turismo colonial se estableció a finales del siglo xIx, primero como un turismo vinculado a la enfermedad. En 1890 aparecen grandes hoteles como el Santa Catalina y el Taoro en el valle de La Orotava (Marrero Henríquez, 2004, p. 18). El atractivo principal son los climas cálidos y los baños de mar. En 1859, Gabriel Belcastel escribe lo siguiente sobre el Puerto de la Cruz, Tenerife:

Cuántas veces hemos bendecido ese inmenso parasol, al tenderse sobre el feliz valle, cubriéndole, en tanto que a lo lejos - dilatándose sobre las olas- nuestros ojos fascinados por la luz, contemplaban su doble centelleo en el azul lejano confundido con el esplendor de los cielos (Gabriel Belcastel, citado en González Lemus, 2007, p. 89).

Esta alabanza a las nubes que cubren el valle de La Orotava no podía mantenerse como un atractivo para el turismo de masas de los años setenta, ochenta y en adelante. El turista colonial se formaría en este contexto, a la luz de las relaciones comerciales de los colonos ingleses (principalmente). El perfil es el de un turista curioso del territorio, de los nativos y de la cultura que visitan. Excelentes reflejos de esa cultura viajera son libros como el de Olivia Stone, Tenerife and its six satellites (1887). Pero esos viajes tenían su propio contexto socioeconómico: las relaciones coloniales iban paralelas a las prácticas turísticas.

Eran también los años de la entrada de la economía canaria en los emporios comerciales extranjeros, especialmente británicos. Desde las primeras décadas del siglo xIX ya estaban operando en las transacciones comerciales isleñas británicos como James Swanston, Robert Houghton, Thomas Miller y otros en Las Palmas de Gran Canaria; y Gilbert Stuart Bruce, Lewis Gellie Hamilton, William Davidson y otros, en Santa Cruz de Tenerife, y a finales del siglo aparecieron hombres de negocios como Henry Wolfson Ossipoff, Richard Ridpath Blandy, Edward Fyffe, Alfred L. Jones, Cecil Barker, Richard J. Yeoward, etc. (González Lemus, 2012, p. 2).

Esta prominente clase colonial no solo impulsó la exportación de mercancías agrícolas para los mercados de las potencias imperiales, sino que además era la responsable directa del auge de este primer turismo colonial, fomentando la creación de los siguientes lugares: la Compañía de Hoteles y Sanatorium del Valle de La Orotava, la Taoro, Compañía de Construcción y Explotación de Hoteles y Villas del Valle de La Orotava, en Tenerife, la Grand Canary Company. Canarias es un territorio generado por prácticas significantes de representación. Como espacio turístico, buena parte de los significantes aprovechados en el proceso dependen de su marco espaciocultural, su significante colonial-exótico. Sin este conjunto de significantes (una cierta distancia, situación geográfica africana, clima cálido y economía terciaria dependiente), Canarias no podría configurarse como un destino. Su imagen es la del sur, pero en un entorno geopolíticamente «seguro" y «estable».

Ese perfil, creado al amparo de las relaciones coloniales, era posible en un contexto anterior a la Segunda Guerra Mundial, en una Canarias marcada por el monocultivo del plátano, introducido por los mismos ingleses, y las divisas extranjeras. En la formación de aquel modelo económico muchos artefactos, palabras y modos de vida se fueron transculturando. 


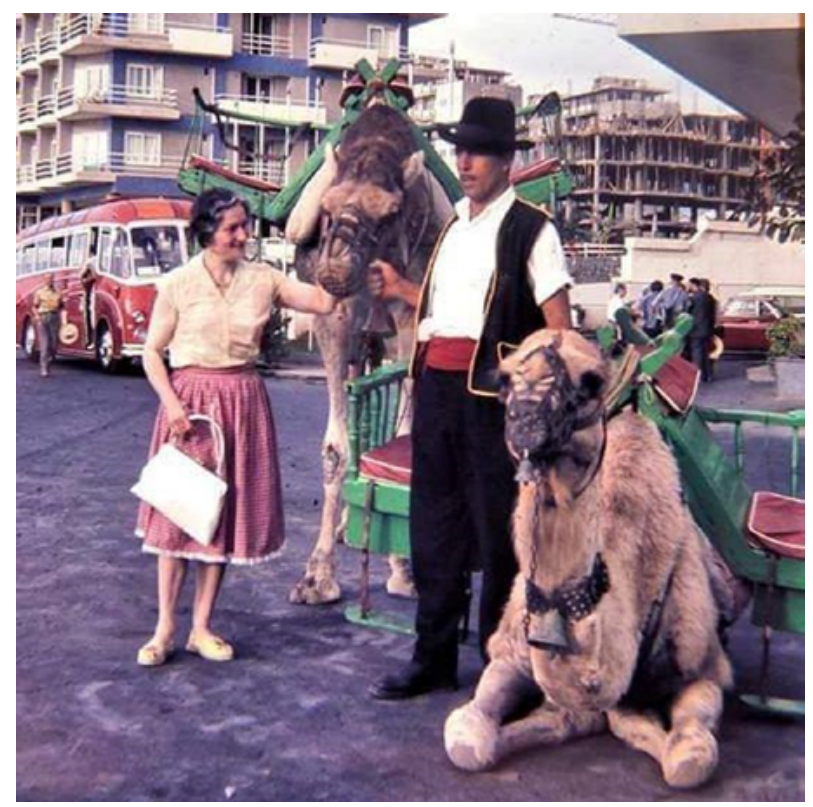

Turista saluda a un camello sujetado por un nativo ataviado al estilo mago, en el Puerto de la Cruz, años sesenta.

Francisco González Díaz, escribiendo en 1910, intentaría intensificar la presencia de este turista colonial, ampliando el perfil para atraer a "los turistas ricos, alegres, despreocupados, mundanos, en buena salud, que viajan por recreo, que gastan y triunfan» (González Díaz en Marrero Henríquez, 2004, p. 19). No le interesan ya los turistas ilustrados, o coloniales, sino la "plebe democrática invasora", porque aquellos «no cifran un contingente positivo de tanta fuerza como las legiones de turistas simplemente curiosos y andarines» (ibid). Se sentaban aquí las bases para el turismo de masas, pero no obstante dominaría aún el tipo de relaciones comerciales y turísticas coloniales, siendo el destino Puerto de la Cruz, en la isla de Tenerife, el lugar ideal por las condiciones climáticas y por la fascinación por la vida cotidiana hacia los nativos del lugar y aledańos. ¿Pero podríamos hablar, en este momento temprano, de un turismo que traspasaba los significantes aplicados en el turismo colonial? ¿Podríamos hablar de turismo de masas?

Mucho/as sociólogo/as del turismo han señalado que el origen del turismo moderno nada tiene que ver con movilidades que se dieron en otros tiempos (especialmente presentan discontinuidades con el viaje colonial y con el Grand Tour). Mantengo, no obstante, que el tipo de prácticas turísticas acontecidas en Canarias responden a una tipología básica de dos perfiles (colonial y poscolonial), pero, pese a la continuidad de una modernidad/colonialidad que permea por ambos periodos, es necesario recurrir a unas coordenadas sociológicas para explicar este salto de per- 
files. Voy a seguir aquí una teoría que sitúa los gérmenes de este turismo de masas en coordenadas más cercanas.

Turismo colonial: aristocrático, viajeros, culto, busca las diferentes culturas, el exotismo y clima estable.

Turismo poscolonial: de masas, de clase media-baja, busca el sol y la playa encontrando una simulación de su lugar de origen. También incluye el posturismo.

De un primer sistema interactivo que tiene que ver con lo resaltado arriba: marcado por la movilidad en barcos y el imperativo del comercio, se dio lugar a las primeras formas de turismo organizado, más allá de una práctica continuada desde el Grand Tour, gracias a la creación de una infraestructura de carretas y el abaratamiento de sistemas de transporte. Como demostró John Urry (1990), el turismo moderno tenía más que ver con el paradigma del Sea side resort, donde las comunicaciones terrestres favorecieron el ocio dentro del entramado de regiones nacionales de la costa en el norte europeo. Este turismo nacional se vio pronto sobrepasado por el internacional, relacionado con la zona del Mediterráneo debido, entre otros factores, al abaratamiento del transporte aéreo y la calidad de las aguas. Fueron estos sistemas (sistema del automóvil, de los trenes, el tráfico aéreo) los que hoy son yuxtapuestos a los sistemas informáticos e internet como una emergente y totalizante infraestructura que sustenta las prácticas turísticas y la movilidad en la actualidad.

Con este giro nace el turismo de masas y con él una nueva geografía que incluye una periferia del placer orbitando alrededor de un centro noreuropeo que empezaba a ser postindustrial. La coincidencia de estos dos fenómenos, unida a los procesos de descolonización del llamado Tercer Mundo, pueden encontrarse en un extraño cuento corto de J.G. Ballard (2002), sobre una estancia en un complejo turístico al sur de Las Palmas de Gran Canaria, que comentaré más abajo. Por ahora, es preciso notar que el perfil del turista poscolonial está cambiando su mirada (Urry, 1990), su manera de entender el mundo, creando una semiótica que es internacional (Culler, 1981) y que permite entender a los turistas como una sociedad global. Estos nuevos encuentros no solo vienen marcados por los pseudoeventos del turismo (encuentros en mundos artificiales y codificados de los hoteles y complejos turísticos), contenidos también por una síntesis de gestión racional y temática como la mcdisneyficación (Ritzer y Liska, 1997), sino que implican aspectos que tienen que ver con la pérdida de identidad en las sociedades industriales y la búsqueda por tanto de experiencias auténticas, aunque estas tienen que estar siempre supeditas a puestas en escena por parte de los nativos, por tanto solo dándose en la relación turística (MacCannell, 2003).

En España, una serie de cambios en la geopolítica tras la reconciliación con EE. UU. propiciaron que el régimen franquista usara el turismo de dos maneras instrumentales: como herramienta comercial y como propaganda de cara al exterior, para demostrar que en España «no se vivía tan mal» (Murray, 2015, p. 199). Iván Murray (2015) dice que el auge del turismo tenía unas razones geopolíticas y económicas que provenían del régimen mismo, no eran fuerzas ajenas a él. No obstante: 
«La temporización del proceso de turistización española se adapta casi perfectamente a las etapas del capitalismo histórico posterior a la II Guerra Mundial» (Murray, 2015, p. 199). Aquí es donde se podrían añadir los procesos de descolonización y los nuevos discursos que imperaron en torno al desarrollismo. Aquí es donde cuadra el perfil del turista poscolonial que, con su mirada (pos)moderna, fue recreando el paisaje que anhelaba, como un espacio marcado por el consumo de masas y por la búsqueda, aún en las reminiscencias de la autenticidad escenificada, de un espacio utópico, contenido por los delineamientos de los enclaves turísticos.

\section{LA ISLA COMO ENCLAVE MONOFUNCIONAL}

Las islas, como dice María Rosa Alonso (1972) en el encabezado de este texto, suponen espacios de alivio en el mar, pero nunca «tierra en serio». Las islas inaccesibles, islas islas, se conforman como espacios del deseo. Si la tierra en serio es el terreno de lo real para las masas de trabajadores, que viven en la normalidad estándar de la vida industrial o postindustrial, en los continentes ricos de este planeta, entonces la isla se convierte en el objeto tentador de su deseo. De esta manera, la isla siempre ha estado, desde Tomás Moro al menos, unida a la idea de utopía. Los mitos clásicos de Canarias siempre giraron en torno a estas consideraciones. Por ello entran en el terreno de la otredad (Minca, 2009, p. 92).

A esto es a lo que Claudio Minca se refiere como el «efecto de la isla», un efecto que provoca todo un imaginario en torno a estos espacios cortados por el mar. En el anverso enfoque del nativo isleño, como en el caso del escritor cubano Virgilio Piñera, la isla es la "maldita circunstancia del agua por todas partes». En uno de los libros más importantes sobre las culturas caribeñas y su espacio atlántico, el también cubano Antonio Benítez Rojo (2001) invita a todas las islas del Atlántico a pensarse como un continente. En su trabajo temprano sobre esta temática (Benítez Rojo, 1998), consideró que las islas del Caribe podían ser tipificadas de manera ideal como una sola isla que se repite y que las incluye a todas. Posteriormente, también consideró que los elementos que había señalado como repetitivos se daban en los archipiélagos situados cerca de la costa africana: la mezcla de poblaciones europea, africana y americana, el carnaval, una historia marcada por corsarios, el sistema de plantación y, de manera novedosa, el turismo. El sistema de plantación no era solo referido a una idea de campo y de agricultura; la plantación es todo un sistema, un campo de concentración, un panóptico, una forma de organización social y política, con su propia moral y su propia fuente de resistencia. Los acoples de la plantación crean, en palabras del autor, un parque de máquinas, que había empezado con aquella "máquina de Colón", pero que proseguía en los intentos de crear ingenios azucareros:

Hubo ciertos hombres de empresa -como el judío Cristóbal de Ponte y el Jarife de Berbería- que intentaron construir modelos de esta familia de máquinas en las Canarias y en el litoral marroquí, pero el negocio era demasiado grande para un solo hombre. En realidad hacía falta todo un reino, una monarquía mercantilista, 
para impulsar los engranajes, molinos y ruedas de esta pesada y compleja máquina. Quiero llegar al hecho de que, a fin de cuentas, fueron las potencias europeas las que controlaron la fabricación, el mantenimiento, la tecnología y la reproducción de las máquinas plantaciones, sobre todo en lo que toca al modelo de producir azúcar de caña (Benítez Rojo, 1998, p. 23. Cursivas añadidas).

Aquí Benítez Rojo está siguiendo la definición que Gilles Deleuze y Félix Guattari (1985) hacen de la máquina como un sistema de acoples y cortes de flujo, para dar con una descomunal máquina-máquina-máquina que ha operado en la creación del Atlántico como ombligo del sistema-mundo moderno colonial capitalista. Pero en su invitación a crear una Nueva Atlántida (tal como míticamente llamó a su conteniente imaginario), Benítez Rojo se da cuenta de que ya no plantamos cańa de azúcar, sino turistas: «lo que ayer fue plantación esclavista [...] hoy es resort turístico» (2001, p. 162). Entonces sí, mantenemos ciertas plantaciones como los plátanos (todo un significante cultural de Canarias), pero «apoyados por plantaciones de hoteles y restaurantes para turistas» (Benítez Rojo, 2001, p. 164).

Se corre el riesgo de restarles importancia a las condiciones esclavistas de la Plantación con estas comparativas, pero creo que hay que tomar la iniciativa, como ya hace Benítez Rojo, para ver las continuidades; no solo de cómo esta área societal estructuró el espacio y dispuso los cuerpos, sino también de ver las resistencias y las formaciones culturales. El turismo no es un mero sector, relacionado con un sistema discreto y seccionado de la cultura isleña; es, más bien, un sistema que abarca todo el espectro social de las Islas. Es el nuevo principio regulador que concentra todos los componentes en la articulación de las sociedades en Canarias. Por lo tanto, siguiendo a Benítez Rojo, podríamos decir que el turismo es también una máquina que se acopla a tantas otras máquinas (creando un nuevo parque): una máquina política, una máquina comercial, una legal, una militar, una estatal, etc.

Esta misma idea también nos acerca a entender el principio regulador del turismo como una forma de ordenar y clasificar los aspectos que entran en juego en las islas. En este sentido, es el investigador Claudio Minca (2009) el que más se ha aproximado, a veces de manera provocadora, a emplear conceptos foucaultianos que definían los regímenes de poder modernos para entender lo que él llama enclaves monofuncionales turisticos. Estos enclaves empezaron a aparecer en Canarias, tras los primeros grandes asentamientos, que, como el Puerto de la Cruz, incorporan la contradicción de ser referentes históricos del turismo al tiempo que ruinas. Clara Muñoz acertó al significar el cambio de sensibilidades del encuentro en este estadio:

Por su tamaño, su especialización en el uso del espacio y su actividad creciente, estos enclaves son los escenarios litorales del Archipiélago con una implantación turística más importante, entornos domesticados mediante una oferta [...] más adecuada para satisfacer las demandas de confort [...] que para propiciar un encuentro antropológico intenso entre visitantes y nativos (Muñoz, 2004, p. 98).

Queda claro que aquí estamos acotando unos espacios segregados que aspiran a dejar fuera todo el ensamble que estoy proyectando, pero por ahora considero preciso entrar a discutir algunas ideas de Claudio Minca. 
En sus estudios sobre la prisión, la clínica y la sexualidad (entre otros), Michel Foucault (véanse 2007 y 2008) fue detallando una teoría de la operatividad del poder que empezaba a tener incidencia en las relaciones espaciales, pero también en la ordenación de los hábitos y las acciones sociales, al tiempo que en el disciplinamiento del cuerpo. Esta nueva operatividad nacía en el siglo XviII, con lo que denominó «el descubrimiento de la población» (Foucault, 2007), que implicaba todo un cambio en las fórmulas del poder soberano estatal. De un pueblo se pasaba a una población (Agamben, 2000, p. 88). En sentido foucaultiano, podemos definir la biopolítica entre dos parámetros clave. Por un lado, está la preocupación por el cuerpo, por disciplinarlo, convirtiéndolo en dócil y útil, algo que llamó «anatomopolítica del cuerpo humano" (Foucault, 2007, p. 168). Por otro, estaba el surgimiento de una abstracción mayor que era entendida dentro de las variables aportadas por la demografía: fecundidad, mortalidad, longevidad, etc. Esto es la biopolítica, que incluye toda una proposición de la delimitación espacial para controlar a los cuerpos (Foucault, 2008). Minca entiende la biopolítica en este sentido amplio, que incluye los dos parámetros como una síntesis de poder. Partiendo de una definición propia de los enclaves turísticos como islas (en el sentido metafórico que alude a su segmentación y al efecto antes descrito), Minca observa que la biopolítica, que se disemina por las instituciones que magistralmente estudió Foucault, también se da en los enclaves ${ }^{8}$ :

How do we explain, then, the fact that many tourist enclaves are regimented in ways similar to those same institutions -both for the workers, and, to some extent, for the tourists themselves? One possible answer is that this partially hidden -but very real- regimentation is not simply about pleasure and money, but also about the biopolitical production of docile subjects and bodies (Minca, 2009, p. 95).

Se podría aquí parafrasear la inversión de Foucault con respecto a la famosa frase del militar Carl von Clausewitz («la política es la guerra por otros medios»): el turismo es la guerra por otros medios?. Cabe recordar que para Foucault, la guerra por otros medios es en sí una guerra sin sujetos; la prolongación de sistemas de control y sujeción que extiende las relaciones de poder. Por supuesto, esta apuesta por estudiar los enclaves turísticos debe mucho al concepto de heterotopía, así como a las geometrías del poder nombradas ya (Minca, 2009, p. 95). Minca se fija en la fuerte reglamentación que tiene el turismo. Pese a que por definición se trata siempre de una cultura del placer, asociada a una semiótica común sobre el disfrute de ver ciertas cosas (Culler, 1981), evadiéndose de las normas sociales de la «tierra firme» y recreándose como anverso al tiempo regulado del trabajo, el turismo supone una cultura ordenada del viaje (Estévez, 2012). Este orden adquiere las dimensiones biopolíticas

8 Siguiendo en la línea de estudios sobre «espacios segregados turísticos», Minca ofrece una literatura parcial pero significativa para lo que estamos tratando aquí. Véase su bibliografía.

${ }^{9}$ Clayton y Korstanje (2012) van más allá al vincular el turismo con la sacralización de espacios tras actos de terrorismo, incidiendo por tanto en el fenómeno y proponiendo que «el turismo es el terrorismo por otros medios". 
que intuye Minca cuando hablamos de los enclaves, sobre todo de espacios aún más reducidos como los hoteles ${ }^{10}$, aunque también se transiten espacios abiertos "programados» (Muñoz, 2004, p. 105). En ellos el cuerpo del turista debe seguir una serie de normas y no estar siempre pasivo, en reposo. Hay un schedule determinado, unas actividades que hacer, e incluso el imperativo de relejarse debe ser visto siempre en un contexto para ello. Lo mismo ocurre para los cuerpos que trabajan en el complejo turístico. Tal y como observa Minca, esto supone una forma de militarización del espacio que afecta a los sujetos que interactúan en él ${ }^{11}$.

Para que esta cultura ordenada pueda darse es necesario que exista una frontera estable. Esto recrea una paradoja: el turista ansía el otro lugar, que por su diferencia ha configurado su deseo, pero la frontera debe restringir la contaminación de los factores externos al complejo turístico. Lo que debe evitar es la presencia nativa (sobre las relaciones de poder entre estos antagonistas y otras figuras intermedias, véase Cheong y Miller, 2000, p. 379), pero también de todo lo que el cuerpo del turista percibe como un riesgo (Korstanje, 2012a). Como veremos más adelante, los riesgos modernos, estudiados por Ulrich Beck, suponen un componente importante en la configuración del destino turístico. Por ahora, es solo necesario saber de la eficacia de esa frontera a la hora de estabilizar la fantasía turística y de protegerla del mundo exterior. Supone añadir que la mirada turística es también una mirada desviada de ciertos aspectos. El giro provocador viene al establecer una serie de paralelismos entre estas formas de regular el viaje y aquella experiencia de los campos de concentración de todo tipo, que se expandieron también en las sociedades de plantación, delineando y segregando el espacio.

The 'Island' regime is indeed an exceptional regime that applies to both tourists and workers, united here by the fact that 'the Island' is regulated by extraterritorial

${ }^{10}$ Esta regulación biopolítica también supone un proyecto de orden contra el desorden propio del caos. En este sentido no deja de ser interesante la apreciación de George Orwell sobre las dos dimensiones del hotel:

Take cleaningness, for example. The dirt in Hotel X, as soon as one penetrated into the services quarters, was revolting. [...] Dirtiness is inherent in hotels and restaurants, because sound food is sacrifice to punctuality and smartness. The hotel employee is too busy getting food ready to remember that it is meant to be eaten. A meal is simple «una comande» to him, just as a man dying of cancer is «a case» to the doctor (George Orwell, Down and out in Paris and London).

${ }^{11}$ Expandiéndose en los ejemplos que da al respecto, Minca observa que:

... the militarization of enclavic tourist spaces takes different, more subtle, forms as well: many resorts feature a semi-military regimentation of the time and the spaces of leisure, with a geometric micro-geography of individuals' (tourists' and workers') movements and activities: 'wake up, be active, follow the club's rules, socialize, rest, have fun, dance, relax, have lunch, but also follow the path to the next attraction, dress in a certain way, never step out of the frontstage, buy, consume, smile, never ask questions, get a new identity, you are just a passenger, a guest, a visitor, a tourist -your body is not yours anymore'. But also, for the worker: 'be silent, be sociable, be visible, be invisible, use the uniform, smile, perform, follow the rules, impose the rules, protect, surveil, surveil, surveil, never forget that you are surveilled!' The entire landscape must emanate a sense of order and control (Minca, 2009, p. 95). 
rules and codes. There is thus one last important similarity between the camp and 'the Island' that deserves our attention, especially if we accept the idea that 'the Island' is the ultimate laboratory for experimenting on how tourist organizations rely, at the same time, on both the control and the metaphorical 'imprisonment' of the body of the worker (and sometime also of the tourist) and on the creativity, the difference, the life-force that emanates from the workers' and the tourists' bodily interactions (Minca, 2009, p. 104).

Pero las sugerentes observaciones de Minca, y el establecimiento de estos paralelismos, apenas nos ofrecen la posibilidad de entender la complejidad con la que los factores biopolíticos se expanden fuera del enclave. Revisaré cuatro aspectos que pueden extenderse teóricamente desde la perspectiva de Canarias como enclave turístico monofuncional. En primer lugar: para Minca, como para Foucault, el espacio es intrínseco a los discursos que los generan, lo que a su vez delimita la operatividad del poder a unas instituciones localizadas, como la prisión o la clínica. En palabras de Jonathan Murdoch (2006, pp. 56-57): «las relaciones de poder parecen estar flotando en una especie de vacío espacial». Para el caso particular de Canarias, los enclaves turísticos no pueden estudiarse como espacios absolutamente cerrados e ideales. Debemos estudiar las lógicas que ahí operan como conectadas; no por una especie de efecto cascada (trickle down), sino como una red de interacción que puede cubrir el territorio de la(s) isla(s). La máquina-máquina deleuziana tiene aquí una aproximación más precisa, pero hay que recordar también que, en islas como Lanzarote, el turismo ha sido un proyecto que marca no solo las políticas en los enclaves, como Puerto del Carmen, sino todo el sistema de carreteras, uniendo inesperadamente el arte de César Manrique con la creación de estos espacios (De Santa Ana, 2016).

En segundo lugar, la frontera no aparece como un tropo que sitúa los espacios del turista solamente, sino como una fuerza articulada en los contornos líquidos de las islas, fijada por los componentes de vigilancia estatal y privado (en una relación humanos/no-humanos) que puede verse idealizada en la tecnología del Sistema Integrado de Vigilancia Exterior $(\mathrm{SIVE})^{12}$. Esto marca no solo la soberanía nacional española, sino el sentido de pertenencia moderna a un lado de la dura línea de la diferencia colonial, una vigilancia que establece también la seguridad del destino y que sigue empujando la ambivalencia de un archipiélago paradisiaco africano de pertenencia europea (como ultraperiferia).

En tercer lugar, las formas de disciplinar a los trabajadores del enclave, actuando certeramente sobre la performance corporal (aspectos que podemos ligar a

${ }^{12}$ Canarias no solo comprende fronteras entre los enclaves y lo local, sino que es, en sí misma, una región fronteriza insular (Godenau y Zapata, 2008, p. 69). Los estudios de frontera (Border Studies) tienen poca repercusión en el ámbito académico canario, pero quizás, para comenzar a articular un sentido crítico del espacio geopolítico canario, deberíamos estudiar en sincronía el significado y el establecimiento de estos dos espacios fronterizos: nacional-europeo y turístico-nativo. Algunos estudios relevantes en este ámbito son Richardson (2013), Pezzani y Heller (2013) y López-Sala (2015), entre otros. 
las apreciaciones de Dean Maccannell sobre la performatividad goffmaniana puesta en marcha en la escena turística), inciden en que el trabajador debe actuar de manera dócil y complaciente:

... is the biopolitical reproduction of a specific smiling and standardized de-personalized body of the worker; a body that is depoliticized (or, alternatively, entirely politicized) to the point that it becomes a manikin, a figurehead, someone who can be seamlessly substituted by someone else-since (s)he is there to perform just a simulacrum of real life (Minca, 2009, p. 100).

El mismo Minca comprende como esta tendencia biopolítica puede extenderse al conjunto de la población:

This form of politicization of performative bodies is even more pervasive when it is imposed outside an 'island-like-resort', when the entire population of a village, for instance, becomes subject to the same logic and expected to 'perform itself', to become just an assemblage of bodies pretending that they are something else, some hypothetical traditional community (Minca, 2009, p. 100).

En la isla de Tenerife, por ejemplo, se propuso en 2003 una campaña publicitaria que con bastante insistencia hacía hincapié en una "Tenerife amable». Funcionaba claramente como una forma de disciplinamiento de la población, aunque todos/as sabemos que en la isla somos a mables por «naturaleza» (disculpen la ironía).

Por último, el enclave como espacio del destino y tipo ideal de la contención humana presenta problemas de método para la compresión de fenómenos antropológicos que se dan en ciertas dimensiones, como la transculturación. Aquí existen una serie de prejuicios para tomar el contacto cultural entre turistas poscoloniales y nativos como una forma de intercambio de información que da pie a tendencias $\mathrm{y}$ formas culturales en el espacio local (y que finalmente no queda restringido a éste). Pese al estudio de formas de transculturación del turismo colonial, existe un vacío académico de los impactos culturales del nuevo turismo, sin llegar a tomarlo como una forma artificial y tendenciosa de afectar la cultura local ${ }^{13}$. Nuevamente es Minca el que provee un concepto para escapar metodológicamente de esta encerrona: el «espacio de lo inesperado»:

This is a space that the capitalist production of leisure tries to manage, tame and possibly reduce to a minimum but, paradoxically, this is also a space without which the endless re-enactment of the tourist performance (a reenactment that constitutes the very foundation of any tourist resort) would simply not take place. Further-

${ }_{13}$ Un ejemplo interesante en este sentido es la transculturación de un particular estilo de música electrónica (llamada hardcore o breakbeat) que provenía de Gran Bretaña (a finales de los ochenta del siglo pasado) y que comenzó a circular por los circuitos de ocio de un enclave turístico en el sur de Tenerife. Rápidamente, los/as nativos/as emularon las fórmulas y desplegaron todo un sentido musical y cultural que se expandió por varias generaciones. 
more, this 'space of the unexpected', as a genuine and ambivalent product of the encounter between the tourist subject and the tourist worker, can never be eliminated or simply reduced to real imprisonment or full control (Minca, 2009, p. 91).

Aun con esta apreciación, las conexiones no se dejan ver en la literatura especializada de los espacios cerrados, al tiempo que la obsesiva atención a los aspectos biopolíticos deja poca cancha para las interacciones a gran escala. La insistencia en el trabajador (masculino), y no en el/la nativo/a, también limita las posibilidades de la crítica feminista, además de la crítica poscolonial o descolonial a la hora de entender el orden racista que reproduce el sistema turístico. Por lo tanto, es una plegaria por el/la nativo/a lo que emerge de la atención a esta literatura, algo que a continuación comentaré junto a un cuento clave sobre el turismo en Canarias.

\section{EL GIRO GEOPOLÍTICO DEL MIEDO}

Pero ante la vorágine de crecimiento asociado a las últimas fases del capitalismo, magistralmente estudiada por Iván Murray (2015), el crecimiento urbano y las extensiones del turismo acabarían por verse subsumidas en el estancamiento de la crisis de 2008. La sorprendente salida de ese escenario fue un inesperado giro geopolítico que revirtió el flujo de movilidad turística hacia Canarias: las oleadas de primaveras árabes en los países del Magreb:

Ante la movilización en los destinos turísticos de los países árabes, los flujos turísticos se empezaron a desviar hacia las playas «tranquilas» de España. De esta manera, los turistas internacionales, que habían caído desde el máximo histórico de 58,6 millones del 2007 a 52,6 millones en el 2010, aumentaron espectacularmente a raíz de las primaveras árabes hasta alcanzar el máximo histórico de 60,6 millones en el 2013 (Murray, 2015, p. 393).

A la luz de esto, parece que los esquemas economicistas distan de ofrecer un análisis que comprenda los eventuales giros geopolíticos como el señalado. La estabilidad de un destino depende en mayor medida de patrones que pueden estudiarse con cierta complejidad, aspirando a situarse dentro de la teoría del caos que guía los sucesos históricos y económicos a escala global. Pero, sin querer presentar una prospectiva, debemos incidir aquí en una forma de organizar el espacio del turismo, pero a una escala global. La apuesta de este procedimiento podría equipararse con una teoría que durante los últimos veinte años ha estado en el centro de la comprensión de un nuevo sistema interactivo global: la teoría del riesgo de Ulrich Beck (2006).

El reparto de riquezas viene acompañado de un reparto sistemático de riesgos, decía Beck. Apuntando a los riesgos globales como aquellos que exceden los riesgos personales y que deben ser mediados por especialistas para ser percibidos como tales, los riesgos modernos globales de los que habla Beck trascienden las fronteras y los rígidos sistemas de control de los Estado-nación, volatilizando también la estructura de clases. Ahora bien, existe un orden jerárquico internacional en el reparto de riesgos que se yuxtapone a una diferencia colonial que, como base del 
racismo moderno, estructura las formas de poder global entre conectados y parias de la modernidad (Bauman, 2005). Aquí es donde resuena de nuevo la posición inestable, fronteriza (controlada por el SIVE) y ambivalente de Canarias. Como destino turístico, su posición en el mercado mundial se establece no solo por el outcome de flujos económicos circulando en un espacio vacío, sino por una geografía movida por el capital y el miedo:

La producción turística del espacio pone en evidencia la polarización socioterritorial. La difusión del capital turístico global, protagonizado básicamente por las ETN [empresas transnacionales], en las periferias del placer se ha materializado territorialmente en enclaves turísticos - resorts, urbanizaciones cerradas (gated communities) o cruceros turísticos-. Estas soluciones espaciales responden, en buena medida, a las retóricas del miedo como ocurre en los espacios urbanos (Blázquez, Cańada y Murray, 2010 sin paginar).

El accidente nuclear de Chernóbil, el clarividente drama de la sociedad del riesgo, fue el constructor colateral del turismo de alquiler vacacional en la isla de $\mathrm{La}$ Palma ${ }^{14}$. Este efecto, tanto como el giro geopolítico dado por las primaveras árabes, nos muestra cómo la geopolítica sigue afectando directamente las relaciones espaciales que luego son complementadas por una biopolítica que, a su vez, excede el espacio como un contenedor cerrado y acotado, desplegando un mapa mucho más caótico e interconectado de los sistemas turísticos.

Este sentido de riesgo, en la teoría de Beck, configura un tipo de política que comúnmente viene asociada a la política turística (véase también Urry, 1995, p. 183); esto es, la política entendida como policy, o como forma de administración y gestión de problemas sin tener que referir la política a un movimiento utópico de cambio, claramente marcada por los riesgos globales frente al referente de política configurada por la contienda ideológica. Pensada en el mismo sentido, la política no es ya la guerra por otros medios, sino el turismo (como problema exponencial), que debe ser medido, acotado y regulado por los mejores actores del management político.

En el resquebrajamiento de la utopía turística, vía los conflictos abiertos por los impactos del turismo (movimientos sociales, ecologismo, visiones de catástrofe), se abre la posibilidad de leer los espacios turísticos del capitalismo tardío como destinos distópicos, marcados por la aceptación de su impacto, por su reconocido capitalismo (no)encantador (Santa Ana, 2004, p. 66) y por aceptar su inconmensurabilidad con los preceptos del equilibrio ecológico. En los años ochenta, paralelamente a la lectura que ofrece Ballard de los complejos turísticos de Gran Canaria, una televisión alemana rueda un reportaje sobre la decadencia del turismo en Canarias. El título del documental alemán era Suicidio en hormigón, la ruina de las Islas Canarias (1990). El destino distópico, como anverso sincrónico de la utopía turística, está situado de lleno en el discurso y la clasificación de esta nueva era que vivimos.

14 Fue en los ochenta cuando se creó el modelo, «cuando aún no existía ni el concepto». Véase, en prensa: «El alquiler vacacional ‘se inventó en la isla’ por huidos de Chernóbil». El Día, 29/8/2018. 
No deja de ser cierto que, pese a que Ballard soltara algo de tinta sobre el Archipiélago, la creencia en un destino distópico no ronda en la mente de los promotores del turismo. Más bien, Canarias quiere verse amparada por su mito más antiguo: un alivio de calma entre un mar de conflictos y catástrofes globales; un lugar donde nunca pasa nada. Un cacho del Edén en medio de un mundo decadente. Pero la huida al vacío de la política nos deja con un espacio claramente demarcado, subsumido en islas fuertemente controladas, vigiladas y militarizadas, que ocultan de manera eficaz las colonialidades que atraviesan su espacio y que contienen los problemas de ese ocultamiento. En el mismo sentido, el ideal del destino oculta que la misma catástrofe ecológica "global» también se experimenta de manera local por todo el territorio.

\section{DESTINO DISTÓPICO: A MODO DE CONCLUSIÓN}

Claudio Minca no ha dicho nada nuevo en su ensayo (2009). A mediados de los años ochenta, en un fascinante cuento corto (Ballard, 2002), el escritor de ciencia ficción J.B. Ballard, famoso por sus distopías contemporáneas que exploraban el «lado oscuro» de la condición humana, contempló el turismo como un fenómeno distópico de concentración y alienación.

En ese cuento relató la estancia de una pareja de británicos en un complejo hotelero al sur de Las Palmas de Gran Canaria. La pareja, al poco tiempo de iniciar su estancia, empieza a notar que están atrapados y que ningún avión sale de vuelta a Gatwick. Esta situación de permanentes vacaciones provoca disímiles efectos y comportamientos en la pareja: ella se adapta y se ajusta a la programación del hotel, entendiendo su oferta cultural como un "minirrenacimiento de las artes», y él tiende a la histeria y la paranoia.

Dentro de este confinamiento (el tipo ideal de biopolítica que Minca estudia), otra cosa se revela con respecto a este turismo de masas: nunca puede obtenerse un contacto directo con lo local o con lo nativo. En una postal, Diane, la protagonista, cuenta: «iTe das cuenta de que Richard y yo hemos estado tan ocupados que en ningún momento nos hemos molestado en visitar Las Palmas?». Luego descubrimos que no solo obedece a un asunto de tiempo u ocupación, sino que es imposible dicho viaje, dicho movimiento hacia lo local. Ante la imposibilidad del viaje, Richard (el supuesto marido de Diane) escapa del complejo, para luego ser encontrado por Diane en la playa tiempo después. El encuentro es narrado de esta manera:

Hoy ha ocurrido un hecho extraordinario: he visto a Richard por primera vez desde que se fue. Yo iba por la playa, practicando mis ejercicios matutinos y allí estaba él, sentado debajo de una sombrilla. Se le veía muy bronceado y saludable, pero mucho más delgado. Con voz tranquila me contó una historia absurda sobre la construcción en todo el territorio de las Canarias, por los gobiernos de Europa occidental en combinación con las autoridades espańolas, de una especie de campo permanente de vacaciones para sus desocupados, no solamente los obreros de las fábricas sino la mayoría del personal de dirección. Según Richard están construyendo una playa para los franceses del otro lado de la isla, y otra para los alemanes. 
Y las Canarias son sólo uno de los muchos lugares alrededor del Mediterráneo y del Caribe. Una vez instalados allí, a los turistas no se les permitirá regresar, por miedo a que inicien revoluciones. Traté de discutir con él, pero se levantó con naturalidad y dijo que iba a formar un grupo de resistencia, y luego se alejó caminando por la playa. El problema es que no ha encontrado nada en que ocupar la mente: ojalá hubiera entrado en nuestro grupo de teatro. Diane.

Es justo en este momento en el que entrevemos la posibilidad de encontrar en el turismo un elemento distópico. Si creemos la versión de Richard, exagerada por la intencionalidad y la conspiración, entonces entendemos el confinamiento que viven como una regulación propia de los campos de concentración. Pero también, si insistimos en la subjetividad de Diane, entendemos el goce experimentado en la alienación que se produce más allá del placer inmediato y fugaz del encuentro. El entramado turístico como normalidad es la paradoja recreada por esta historia que nos muestra que toda nuestra sociedad se ha turistificado. Estamos ante una sociedad del espectáculo: si la aceptas, sobrevives como Diane; si no la aceptas, la resistencia se convierte en paranoia y desolación, como le ocurre a Richard. ¿Qué lectura pueden hacer los nativos de esta paradoja, en un cuento donde están ausentes? ¿Qué significa vivir la normalidad en lugares creados para excederla; no-lugares donde el significado nunca puede estar fijado por estar atravesado por movilidades?

Si el turismo es la guerra por otros medios, entonces la gente nativa tiene una posición determinada en la configuración de este espacio. En distintos enclaves turísticos, el sentido aséptico de la política turística, claramente entendida como una forma de política sin política (tan solo como gestión administrativa moderna de una serie de recursos y problemas), pronto se ve replanteada como un campo de lucha por los valores identitarios, puestos en peligro por el turismo poscolonial de masas. El turismo queda entonces dividido como en un escenario de guerra e invasión (metáforas habituales en el terreno) ${ }^{15}$, donde la excepción a la norma es la norma. Reflexionando sobre el turismo como una última forma de mitología implantada en Canarias, Larisa Pérez Flores se pregunta: “¿Cómo enfrentar tamaña red mitológica desde una reconstrucción identitaria? ¿Cómo llenarla de contenido para poner freno a la depredación de los bienes naturales y culturales que implica la economía neocolonial?» (Pérez Flores, 2018, p. 14).

Recibido: 1 de junio de 2021; Aceptado: 29 de julio de 2021

15 Entre algunas de las consecuencias de la máquina turística capitalista en Canarias está el impacto medioambiental y la construcción en la costa, ambos relacionados con el llamado tsunami urbanístico y el boom inmobiliario que acabaría con la crisis económica global de 2008. Canarias acabaría haciendo artificial buena parte de su costa (Murray, 2015, p. 358). 


\section{BIBLIOGRAFÍA}

Agamben, G. (2000). Homo sacer III. Lo que queda de Auschwitz, el archivo y el testigo. Pre-textos.

Alonso, M.R. (1972). Papeles tinerfeños. Ediciones Nuestro Arte.

Ballard J.G. (2002). «Días maravillosos», en Mitos del futuro próximo. Minotauro.

Barrado-Timón, D. (2004). «El concepto de destino turístico. Una aproximación geográfico-territorial». Estudios Turísticos, núm. 160, pp. 45-68.

Bauman, Z. (2005). Vidas desperdiciadas, la modernidad y sus parias. Paídos.

Веск, U. (2006). La sociedad del riesgo. Hacia una nueva modernidad. Paídos.

Benítez Rojo, A. (1998). La isla que se repite. El Caribe y la perspectiva posmoderna. Casiopea.

Benítez Rojo, A. (2001). «Nueva Atlantida. ¿Un futuro continente?» Revista La Tadeo, n. ${ }^{\circ} 66$. Segundo semestre.

Blázquez, M., Cañada, E. y Murray, I. (2010). «Búnker playa-sol. Conflictos derivados de la construcción de enclaves de capital transnacional turístico español en El Caribe y Centroamérica». Scripta Nova. Revista electrónica de geografía y ciencias sociales, vol. xv, n. ${ }^{\circ} 368$.

Castro-Gómez, S. y Grosfoguel, R. (2007). El giro decolonial. Reflexiones para una diversidad epistémica más allá del capitalismo global. Siglo del Hombre Editores.

Cheong, So-Min y Miller, M.L. (2000). «Power and tourism. A Foucauldian Observation». Annals of Tourism Research, vol. 27, n. ${ }^{\circ}$ 2, pp. 371-390.

Clayton, A. y Korstanje, M. (2012). «Turismo: el terrorismo por otros medios». Revista Hospitalidade. São Paulo, vol. Ix, n. ${ }^{\circ}$ 1, pp. 53-81.

Culler, J. (1988). «The Semoitics of Tourism», en Framing the Sign: Criticism and its Institutions. University of Oklahoma Press, Norman.

Deleuze, G. y Guattari, F. (1985). El anti-Edipo. Capitalismo y esquizofrenia. Paídos.

Equipo Neura (1974). Crónica histérica. Película de producción independiente, grabada en súper 8.

Estévez GonzÁLEZ, F. (2007). «Curiosidades, especímenes, souvenirs. Las momias como objetos-viajeros en el tráfico Canarias-Europa», en Oliver Frade, J.M. y Relancio Menéndez, A. (eds.). El descubrimiento cientifico de las Islas Canarias, La Orotava: Fundación Canaria Orotava de Historia de la Ciencia.

Estévez GonzÁlez, F. (2012). «El turista: de flaneur a choraster». FLUOR. n. ${ }^{\circ}$ 3, pp. 30-34.

Foucault, M. (2007). Historia de la sexualidad. 1-La voluntad de saber. Siglo XXI.

Foucault, M. (2008). Vigilar y castigar. El nacimiento de la prisión. Siglo XXI.

Gil Hernández, R. (2015). Los guanches. Conquista y anticonquista del archipiélago canario. Tesis inédita.

Godenau, D. y Zapata V (2008). Canarias: inmigración en una región fronteriza del sur de la Unión Europea. Politica y Sociedad, vol. 45, n. ${ }^{\circ}$, pp. 61-83.

González Lemus, N. (2007). Clima y medicina. Los origenes del turismo en Canarias. IDEA.

González Lemus, N. (2012). «El despegue del turismo en Canarias. El Puerto de la Cruz y el turismo británico de salud». Turismo. Escuela Universitaria Iriarte, vols. 3-4, 2011-2012. pp. 1-11.

Harvey, D. (2000). Spaces of Hope. University of California Press. 
Korstanje, M. (2012). «Los riesgos y el sistema turístico: una nueva aproximación». Anuario Turismo y Sociedad, vol. XIII, pp. 99-116.

MacCannell, D. (2003). El Turista: Una nueva teoría de la clase ociosa. Melusina.

MacCannell, D. (2007). Lugares de encuentro vacios. Melusina.

Marrero Henríquez, J.M. (2004). «Del turista textual, al lector ecológico», en Mariano de Santa Ana (ed.) Paisajes del placer, paisajes de la crisis. El espacio turístico canario y sus representaciones. Fundación César Manrique.

Minca, C. (2009). «The Island, Work, Tourism and the Biopolitical». Tourist Studies. 9 (2). pp. 88-108.

Muñoz, C. (2004). «Seguro de sol», en Santa Ana, Mariano de (ed.): Paisajes del placer, paisajes de la crisis. El espacio turistico canario y sus representaciones. Fundación César Manrique.

Murdoch, J. (2006). Post-structuralist geography. SAGE.

Murray Mas, I. (2015). Capitalismo y turismo en España: del "milagro económico" a la "gran crisis». Editorial Alba Sud.

López Sala, A. (2015). «Exploring Dissuasion as a (Geo)Political Instrument in Irregular Migration Control at the Southern Spanish Maritime Border». Geopolitics. 20, pp. 513-534.

Pérez Flores, L. (2017). Islas, cuerpos y desplazamientos. Las Antillas, Canarias y la descolonización del pensamiento. Tesis inédita. Universidad de La Laguna.

Pérez Flores, L. (2018). Islas, migración y criollización: Canarias desde un enfoque descolonial. Anuario de Estudios Atlánticos, n. ${ }^{\circ}$ 65: 065-021, pp. 1-19.

Pezzani, L. y Heller, C. (2013). «A disobedient gaze: strategic interventions in the knowledge(s) of maritime borders». Postcolonial Studies, vol. 16, n. ${ }^{\circ}$ 3, pp. 289-298.

Pratt, M.L. (1996). «Apocalipsis en los Andes: zonas de contacto y lucha por el poder interpretativo». Conferencia: Banco Interamericano de Desarrollo en Washington, D.C., el 29 de marzo de 1996, en el marco del Programa de Conferencias del Centro Cultural del BID.

Richardson, T. (2013). «Borders and Mobilities: Introduction to the Special Issue». Mobilities, vol. 8, n. ${ }^{\circ} 1$, pp. 1-6.

Ritzer, G. y Liska, A. (1997). «McDisneyization’ and 'Post-Tourism’: Complementary Perspectives on Contemporary Tourism", en Rojek, C. y Urry, J. (eds.): Touring cultures: transformations of travel and theory. London and New York: Routledge.

Rothman, H. (1998). Devil's Bargains: Tourism in the Twentieth-Century American West. University of Kansas Press.

Santa Ana, M. (ed.) (2004). Paisajes del placer, paisajes de la crisis. El espacio turístico canario y sus representaciones. Fundación César Manrique.

Santa Ana, M. (2016). "César Manrique: la obra de arte turístico total». Concreta, n. ${ }^{\circ}$ 10, pp. 16-29.

Thrift, N. (2008). Non-Representational Theory: Space, Politics, Affect. Routledge.

Urry, J. (1990). The Tourist Gaze. Leisure and Travel in Contemporary Societies. SAGE.

URrY, J. (1995). Consuming Places. Routledge.

Welsh, I. (2009.) Si te gusto la escuela, te encantará el trabajo. Anagrama. 